\title{
Time dependent thermal lensing measurements of $V-T$ energy transfer from highly excited $\mathrm{NO}_{2}$
}

\author{
Beatriz M. Toselli, ${ }^{\text {a) }}$ Theresa L. Walunas, ${ }^{\text {b) }}$ and John R. Barker ${ }^{\text {c) }}$ \\ Department of Atmospheric, Oceanic, and Space Sciences, Space Physics Research Laboratory, \\ The University of Michigan, Ann Arbor, Michigan 48109-2143
}

(Received 27 November 1989; accepted 10 January 1990)

\begin{abstract}
The time dependent thermal lensing technique has been used to measure the vibrational relaxation of $\mathrm{NO}_{2}$ (initially excited at $21631 \mathrm{~cm}^{-1}$ ) by $\mathrm{Ar}, \mathrm{Kr}$, and Xe. The energy transfer analysis was carried out in terms of $\langle\langle\Delta E\rangle\rangle$, the bulk average energy transferred per collision. This quantity was found to have a very strong dependence on vibrational energy, with a marked increase at energies greater than about $10000 \mathrm{~cm}^{-1}$, where several electronic excited states $\left({ }^{2} B_{2},{ }^{2} B_{1}\right.$, and $\left.{ }^{2} A_{2}\right)$ mix with the ground state $\left({ }^{2} A_{1}\right)$. This effect may be due to large amplitude vibrational motions associated with the coupled electronic states. Even at low energies, deactivation is faster than in other triatomic systems, probably because $\mathrm{NO}_{2}$ is an open shell molecule and electronic curve crossings provide efficient pathways for vibrational deactivation. The $V-T$ rate constant for deactivation of $\mathrm{NO}_{2}(010)$ by argon is estimated to be $(5.1 \pm 1.0) \times 10^{-14} \mathrm{~cm}^{3} \mathrm{~s}^{-1}$. Results obtained for $\mathrm{NO}_{2}^{*}-\mathrm{NO}_{2}$ collisions gave $\langle\langle\Delta E\rangle\rangle$ values in good agreement with literature results from fluorescence quenching experiments, indicating that $V-T$ may be more important than $V-V$ energy transfer in the quenching process.
\end{abstract}

\section{INTRODUCTION}

Energy transfer in highly excited molecules can be studied using several physical techniques, instead of the traditional unimolecular reaction systems. The physical techniques are advantageous, because they avoid complications due to chemical reaction mechanisms and they can be applied at energies lower than reaction thresholds. Most physical techniques depend crucially on accurate calibrations, which are confined to the specific system studied. In our laboratory, time-resolved infrared fluorescence has been used to measure energy transfer involving azulene, ${ }^{1,2} 112-$ trifluoro ethane, ${ }^{3}$ and benzene ${ }^{4}$ molecules, after laser excitation. Troe and coworkers have used time-resolved ultraviolet absorption to study energy transfer in large polyatomic molecules, ${ }^{5,6} \mathrm{CF}_{3} \mathrm{I}^{7}$ and the triatomics $\mathrm{SO}_{2}{ }^{8}$ and $\mathrm{CS}_{2} .{ }^{9} \mathrm{~A}$ weakness of these methods is that the calibration is very important and must be carried out for each separate system studied. Spectroscopic techniques also require detailed spectroscopic data for each system studied. For example, infrared fluorescence from $\mathrm{NO}_{2}$ excited at $400-500 \mathrm{~nm}$ recently was used to determine the quenching rate constants for deactivation by $\mathrm{NO}_{2}$ and several other gases. ${ }^{10}$

An alternative to physical techniques that require individual calibrations are photothermal techniques, which depend almost exclusively on laser beam characteristics and on the properties of the collider gas, rather than those of the excited molecule. Such techniques include time-dependent thermal lensing (TDTL), ${ }^{11-14}$ time-resolved optoacoustics, ${ }^{13,15} \mathrm{UV}$ absorption by a tracer gas, ${ }^{16}$ and the probe beam deflection technique. ${ }^{17}$

In this paper we present TDTL measurements of $V-T$ energy transfer in $\mathrm{NO}_{2}$ initially excited at $21631 \mathrm{~cm}^{-1}$, in

\footnotetext{
" Postdoctoral research associate

b) Participant in the NSF Research Experience for Undergraduates program.

- Also Department of Chemistry
}

the presence of $\mathrm{Ar}, \mathrm{Kr}$, and $\mathrm{Xe} . \mathrm{NO}_{2}$ has been the object of innumerable studies, partly because it is one of the few chemically stable gaseous species of unpaired electronic spin, and partly because of its strong and complex absorption in the visible. ${ }^{18}$ The notorious complexity of the visible spectrum of $\mathrm{NO}_{2}$ has been attributed to strong perturbations of the electronic excited states $\left({ }^{2} B_{2},{ }^{2} B_{1}\right)$ by vibronic coupling with high-lying levels of the electronic ground state $\left({ }^{2} A_{1}\right)$. $\mathrm{NO}_{2}, \mathrm{SO}_{2}$, and $\mathrm{CS}_{2}$ are members of a class of molecules with overlapping electronic states, which exhibit much longer radiative lifetimes than would be expected from their measured integrated absorption coefficients, as was first discussed by Douglas. ${ }^{19}$

Despite the large number of studies involving the $\mathrm{NO}_{2}$ molecule (which cannot be reviewed here) the understanding of energy transfer in this system is far from complete. Although the visible fluorescence of $\mathrm{NO}_{2}$ has been studied extensively by Kaufman and coworkers, ${ }^{20}$ and information has been obtained about the vibrational quenching rates for $\mathrm{NO}_{2}$ at high levels of excitation, there is only one paper reporting direct measurements of energy transfer that may apply to lower energies, and the interpretation of those results is not straightforward. ${ }^{10}$

The main goal of the present work is to elucidate collisional deactivation processes in $\mathrm{NO}_{2}$ at intermediate and low energies. The TDTL technique was employed because it monitors the energy transfer to the translational degrees of freedom and is independent of the extremely complex spectroscopy of $\mathrm{NO}_{2}$. Furthermore, the technique can be extended to other excited molecules, without the necessity of carrying out additional calibrations. Despite some limitations of the technique, which are described in detail, considerable information was obtained about the $\mathrm{NO}_{2}$ system.

\section{EXPERIMENT}

$\mathrm{NO}_{2}$ (Matheson) was stored in a blackened container after it was purified by mixing with $\mathrm{O}_{2}$ overnight, followed 
by freezing at dry ice temperature and pumping to eliminate $\mathrm{O}_{2}$ and other volatile impurities, until the solid was completely white. The rare gas diluents were cylinder gases of research grade and were used without further purification. All the experiments were carried out at room temperature.

The $\mathrm{NO}_{2}$ molecules absorb some of the energy from the pulsed laser beam and then the absorbed energy is transferred by collision to the translational and rotational degrees of freedom as heat. This energy deposition results in a temperature rise and concomitant reduction in gas density at the center of the beam, but less change away from the center. The change in the gas density produces a variation in the refractive index (a lens), which dissipates at longer times due to thermal conductivity. The sign and magnitude of the time dependent lensing effect are measured by using a pinhole and photomultiplier to monitor the light intensity at the center of a CW He-Ne laser beam, that is counter-propagated with the pump laser. A schematic of the optical setup is shown in Fig. 1. The counter-propagating geometry was chosen to reduce the effects of scattered light from the pump laser, which is many times more intense than the probe laser. Although this geometry is very helpful in reducing interference from the pump laser, accurate coaxial alignment of the two laser beams is nontrivial.

Descriptions of many experimental details can be found in Ref. 12 and 13 . Visible light $(462.3 \mathrm{~nm})$ from the pulsed tunable dye laser (Lumonics Hyperdye-300, using Coumarin 460 dye) pumped by a $\mathrm{XeCl}$ excimer laser (Lumonics Hyperex 400) was directed through a cell containing a mixture of $\mathrm{NO}_{2}$ and a buffer gas (usual ratio of 1:5000). The energy of the dye laser (typically $3 \mathrm{~mJ}$ pulse $^{-1}$; see Table II) was continuously monitored with a Scientech absorbing calorimeter power meter at the position shown in Fig. 1. For a typical pulse length of $\sim 20 \mathrm{~ns}$, this energy corresponds to $\sim 150 \mathrm{~kW}$, compared with the $\sim 0.5 \mathrm{~mW} \mathrm{He} / \mathrm{Ne}$ probe laser (Spectra-Physics, Model 155A). Scattered light from the probe laser was virtually eliminated by filtering with a 6328 $\AA$ interference filter and using a $0.5 \mathrm{~m}$ Ebert monochromator equipped with a R955HA photomultiplier.

The laser beams were combined and separated on quartz-flat beam splitters. To aid in optical alignment, two irises were placed as shown in Fig. 1. After alignment was complete in each experimental run, the irises were opened wide in order to avoid spurious effects due to Fresnel diffraction.

For the pump laser, a well-defined Gaussian beam is desirable, because the initial spatial distribution of the excit-

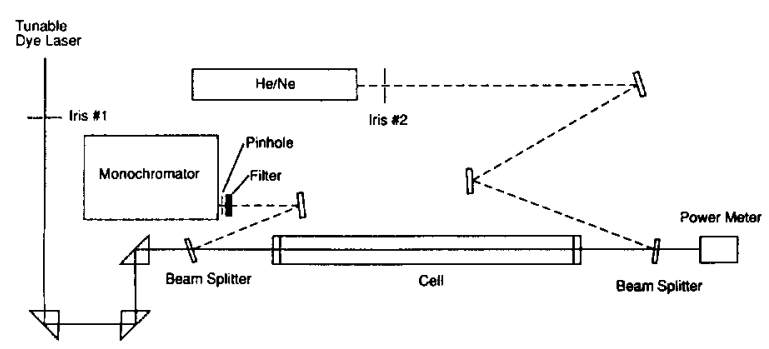

FIG. 1. Experimental apparatus used in the TDTL technique. ed molecules depends on the pump laser beam spatial profile and the theory of the TDTL experiment is most easily applied for Gaussian profiles. Even if the pump laser beam is not Gaussian, the theory can be applied to arbitrary profiles, as long as they are axially symmetric. Unfortunately, the pump laser (Lumonics Hyperdye-300) used in the experiments is pumped through a side window and the beam profile is neither Gaussian, nor axially symmetric, but it has a somewhat triangular to rectangular cross section. The intensity profile of the beam was determined by scanning the beam with a pinhole and photodiode. The beam profile showed an approximately Gaussian shape, but with some superimposed structure and some satellite peaks near the wings. These irregularities can affect the initial TDTL signal shape, but will be gradually eliminated due to thermal conductivity and diffusion of excited molecules in the course of time. As shown below, the theory (based on Gaussian profiles) and experiments showed small differences during the first 5-10 $\mu \mathrm{s}$, but the discrepancies were minor and did not affect the energy transfer results.

The time dependent signals were amplified with a Tektronix AM 502 dc-coupled amplifier and averaged with a LeCroy 9400 digital oscilloscope for 10000-50 000 laser pulses in each experiment. The signal was further analyzed after transfer to a Macintosh personal computer. TDTL signals were obtained as a function of the inert gas pressure from 10 to 500 Torr and simulated using a theory that incorporates all the gas-dynamics factors that affect the density in the system. A brief outline of the theory is presented in the next section.

\section{TDTL THEORY}

The theory of TDTL has been discussed by Flynn and coworkers, " by Bailey and coworkers, ${ }^{21}$ by Barker and coworkers, ${ }^{12,13}$ and recently by Jacobs, ${ }^{22}$ who has presented an accurate numerical method for solving the governing equations for this photothermal system. The following presentation of the TDTL theory is based on the review article from Ref. 13, and the reader should consult that reference and the articles cited there for more details.

After the absorption of the laser radiation the gas-dynamic factors that affect the TDTL signal are: 1) the energydeposition rate to produce translational heat, 2 ) the acoustic waves generated by the heat deposition, 3 ) the binary diffusion of the excited molecules through the bath gas, 4) the thermal conductivity, and 5) the viscosity. In the following analysis, viscosity has been neglected, because its effect is unimportant, as shown by Jacobs. ${ }^{22}$ The magnitude of the thermal lensing effect depends on the competition between the rate of creation of the lens due to $V-T$ energy transfer and the rate of decay of the lens due to the gas-dynamic factors. It is useful to consider the time scales associated with each of these processes.

The time scale for $V-T$ energy transfer can be defined considering a two level system

$$
A^{*}+M \rightarrow A+M \text {, }
$$

where $A^{*}$ is the excited molecule and $M$ is the collider gas. The heat deposition process is characterized by the time con- 
stant

$$
\tau_{e}=\left(k_{1}[M]\right)^{-1}=k_{e}^{-1},
$$

where $k_{1}$ is the bimolecular rate constant, $k_{e}$ is the corresponding pseudo-first order rate constant, and $[M]$ is the collider gas concentration.

For acoustic waves the acoustic transit time across the laser beam is

$$
\tau_{s}=\frac{r_{b}}{c},
$$

where $c$ is the speed of sound and $r_{b}$ is a parameter that characterizes the radius of the pump-laser beam and is very important in controlling the shape of the TDTL signal, as discussed below.

The characteristic time scale for diffusion of excited species is

$$
\tau_{d}=\frac{r_{b}^{2}}{4 D_{12}}
$$

where $D_{12}$ is the binary diffusion coefficient.

According to Bailey et al. ${ }^{21}$ the time scale for thermal conductivity is

$$
\tau_{t}=\frac{P C_{p} r_{b}^{2}}{4 R T \lambda}
$$

where $P$ is the total pressure, $C_{p}$ is the molar heat capacity at constant pressure, $R$ is the gas law constant, $T$ is temperature, and $\lambda$ is the thermal conductivity coefficient.

In energy transfer studies, it is desirable that the energy transfer time scale be well separated from the others, leading to the following idealized guidelines:

$$
\tau_{t}, \tau_{d}>10 \tau_{e}>100 \tau_{s} .
$$

For most polyatomic systems of interest, however, the conditions given by Eq. (6) are rarely met and the only way to extract the rate constant for energy transfer from the TDTL experiments is by comparisons with simulations that take into account all the gas-dynamic processes. This is the approach followed in the present work.

The transient lensing signal $S$ is defined as

$$
S=1-I / I_{o} \text {, }
$$

where $I_{o}$ is the intensity at the center of the unperturbed $\mathrm{He}-$ Ne laser probe laser beam at the position of the pinhole, and $I$ is the corresponding time dependent intensity when the lens is present. For small signals $(<15 \%)$ and when the pinhole radius is much smaller than the laser beam radius, it has been shown ${ }^{21}$ that

$$
S=\left(\frac{Z^{2}}{n_{0}}\right)\left(\frac{\partial^{2} n}{\partial r^{2}}\right)_{r=0},
$$

where $n_{o}$ and $n$ are the refractive indices of the unperturbed and perturbed gas, respectively, and $r$ is the radial distance from the optical axis. For a large signal and for the case when the pinhole is located some distance from the exit of the cell, Eq. (8) becomes ${ }^{13}$

$$
\begin{array}{ll}
S=1-\left[\cosh \left(\xi^{1 / 2} Z\right)+\xi^{1 / 2} L \sinh \left(\xi^{1 / 2} Z\right)\right]^{-2} & \begin{array}{l}
\xi \\
0,
\end{array} \\
S=1-\left[\cos \left(|\xi|^{1 / 2} Z\right)-|\xi|^{1 / 2} L \sin \left(|\xi|^{1 / 2} Z\right)\right]^{-2} & \xi<0 .
\end{array}
$$

[Note that Eqs. (9a) and (9b) differ by a factor $\xi^{1 / 2}$ from that in Ref. 13, where this factor was inadvertently omitted.] In these equations, $Z$ represents the cell length and $L$ is the distance from the exit of the cell to the pinhole. When $L$ can be neglected, these expressions reduce to

$$
\begin{array}{ll}
S=\left[\tanh \left(\xi^{1 / 2} Z\right)\right]^{2} & \xi>0 \\
S=\left[\tan \left(|\xi|^{1 / 2} Z\right)\right]^{2} & \xi<0,
\end{array}
$$

where

$$
\xi=\frac{1}{n_{0}}\left(\frac{\partial^{2} n}{\partial r^{2}}\right)_{r=0} .
$$

The simulations we present in this paper were carried out using Eq. 10. For our experimental conditions, the shape of the signal, and therefore the energy transfer parameters we extracted, were not affected by the sinh term in Eq. 9 as confirmed by direct calculation. However, note that for large signals ( $>0.5$ ) and/or for very large $L$, the sinh term will affect not only the magnitude of the signal but its shape.

The refractive index is related to the gas density $(\rho)$ by the Lorentz-Lorenz law, ${ }^{23}$ leading to the final expression for the TDTL signal:

$$
S=\tanh \left\{\left[\frac{3 A}{2 M}\left(\frac{\partial^{2} \rho}{\partial r^{2}}\right)_{r=0}\right]^{1 / 2} Z\right\}^{2},
$$

where $A$ is the molar refractivity and $M$ is the molecular weight of the gas. The density fluctuation due to energy transfer can be obtained by solving numerically the gas dynamic equations for mass, energy, and momentum. These equations are combined, linearized, and manipulated to produce a third-order partial differential equation for the density as a function of position and time. ${ }^{12,13}$ By expanding the density in terms of zero-order Bessel functions (for convenience in obtaining a tractable solution ), the partial differential equation is transformed to an ordinary differential equation which can be solved by Laplace transforms, using the initial conditions. ${ }^{12,13}$ As pointed out by Jacobs, ${ }^{22}$ use of the zero-order Bessel functions imposes incorrect boundary conditions, leading to a phase-error in the calculated reflected acoustic waves. Except for this deficiency, the Laplace transform solution gives very accurate results and is very computationally efficient.

The original theory derived in Refs. 12 and 13 assumes that the rate constant for $V-T$ energy transfer is a constant. For a two-state system, this will be true, but for a molecule with many vibrational states, the effective rate constant is expected to be a function of vibrational energy, which changes as a function of time. For molecules with high vibrational state densities, elementary $V-T$ rate constants are rarely accessible and it becomes more practical to describe energy transfer in terms of the average amount of energy transferred per collision. When averaged over the population distribution, which is evolving with time, it is necessary to discuss the time-dependent bulk average energy transferred per collision: $\langle\langle\Delta E(t)\rangle\rangle$. This quantity, in a multiple state system undergoing an energy cascade, can be related to an effective $V-T$ rate constant in an effective two-state system (appropriate for the theory), as follows.

The TDTL signal is sensitive only to the energy deposited in the translational degrees of freedom. For a monatomic 
collider gas, the rate of translational energy deposition can be related to the average rate of loss of vibrational energy:

$$
\frac{d q}{d t}=-\frac{d}{d t}\langle\langle E\rangle\rangle
$$

In the two-state system, the rate of energy loss from the ensemble of excited molecules is

$$
\frac{d}{d t}\langle\langle E\rangle\rangle=H_{1} \frac{d}{d t} N_{1}=-H_{1} k_{e} N_{1},
$$

where $\langle\langle E\rangle\rangle$ is the bulk average energy, $N_{1}$ is the number density of excited molecules, $H_{1}$ is the difference in enthalpy between the upper and lower states, and $k_{e}$ is the pseudo-first order rate constant for energy transfer in Eq. (2). Since the excitation energy in the ensemble of excited molecules is just $\langle\langle E\rangle\rangle=H_{1} N_{1}$, Eq. (14) can be written

$$
\frac{d}{d t}\langle\langle E\rangle\rangle=-k_{e}\langle\langle E\rangle\rangle .
$$

By definition, the rate of change of $\langle\langle E\rangle\rangle$ is given in terms of $\langle\langle\Delta E\rangle\rangle$ by

$$
\frac{d}{d t}\langle\langle E\rangle\rangle=k_{\mathrm{L}} N_{c}\langle\langle\Delta E(t)\rangle\rangle,
$$

where $k_{\mathrm{LJ}}$ is the bimolecular rate constant for collisions (assuming Lennard-Jones potentials) and $N_{c}$ is the collider gas number density. Thus, the rate constant for energy transfer is expected to be time-dependent:

$$
k_{e}(t)=-k_{\mathrm{LJ}} N_{c} \frac{\langle\langle\Delta E(t)\rangle\rangle}{\langle\langle E(t)\rangle\rangle} .
$$

In the simulations, a functional form must be assumed for $\langle\langle\Delta E(t)\rangle\rangle$, or for $k_{e}(t)$. Furthermore, the excitation energy $\langle\langle E(t)\rangle\rangle$ residing in the excited molecules at any time can be calculated by numerically integrating Eq. (15). Thus, $\langle\langle\Delta E\rangle\rangle$ can be expressed as a function of the corresponding energy $\langle\langle E\rangle\rangle$. For modeling the data, it was convenient to assume $k_{e}(t)$ is given by the empirical expression:

$$
k_{e}=k_{o} \exp (-A \omega t)+k_{1} \exp (-B \omega t)+k_{\text {lim }},
$$

where $\omega=k_{\mathrm{LJ}} N_{c}$ is the collision frequency. The parameters $k_{o}, A, k_{1}, B$, and $k_{\mathrm{lim}}$ are adjusted empirically to obtain agreement between the simulations and experiments. Equation (18) was found to be the simplest expression adequate to simulate the experimental data. Note that Eq. (18) permits analytical integration of Eq. (15).

The TDTL theory described above can applied for any arbitrary time interval, if it is assumed that $k_{e}$ is constant during the interval and if the initial conditions for the interval are accounted for properly. The only difference from the original theory is that now for each time step the derivative of the coefficients for the Bessel function expansion of the density fluctuation must be evaluated in order to advance the solution. At time zero, the derivatives are equal to zero, but at later times, the spatial distribution evolves and the derivatives vary with time. The results of this approach were compared with the exact solution using the program DIFCOLL kindly provided to us by S. Jacobs, ${ }^{22}$ and both treatments produce exactly the same results (except for the reflected acoustic waves), when sufficiently small time steps are taken.
For comparison with the experimental data, the instrument response function, which depends on the electronics and photomultiplier load resistor, was numerically convolved with the theoretical calculations. For the detailed comparisons with experimental data near the initial acoustic wave spike, this step was necessary, because the electronics introduced small delay times and the photomultiplier time response (with $1200 \Omega$ termination) was about $0.1 \mu \mathrm{s}$.

\section{TDTL SIGNALS}

It is interesting to analyze TDTL signals to determine how they are affected by energy transfer, acoustic waves, diffusion, and thermal conductivity. In Fig. 2 are shown TDTL signals for a mixture of 0.04 Torr of $\mathrm{NO}_{2}$ and 200 Torr of Ar, Kr, and Xe (See Table I for the physical properties of the gases). Note that despite the similarities of the signals, each shows the effects of atomic mass on the thermal conductivity and diffusion coefficients (Table I). The magnitude of the signal under the same conditions depends on the optical properties of each inert gas, especially the molar refractivity, which explains why the signal for $\mathrm{Xe}$ is largest.

The optical and physical properties of the collider gases are very important in determining the feasibility of the TDTL technique for energy transfer measurements, in addition to the properties of the excited molecule under study. For example, $\mathrm{He}$ and $\mathrm{Ne}$ thermal conductivities are so large that thermal conduction is the most important factor controlling the signal at all times, and energy transfer could not be measured. Moreover, the low molar refractivities of these gases produced small signal amplitudes and usable signals could not be obtained at the high dilution ratios necessary.

The initial rising portion of the signals is controlled mostly by the rate of energy transfer and by acoustic waves, while the decaying portion is dominated by thermal conductivity. Diffusion plays a small role throughout. The initial spike observed in all of the signals occurs because part of the

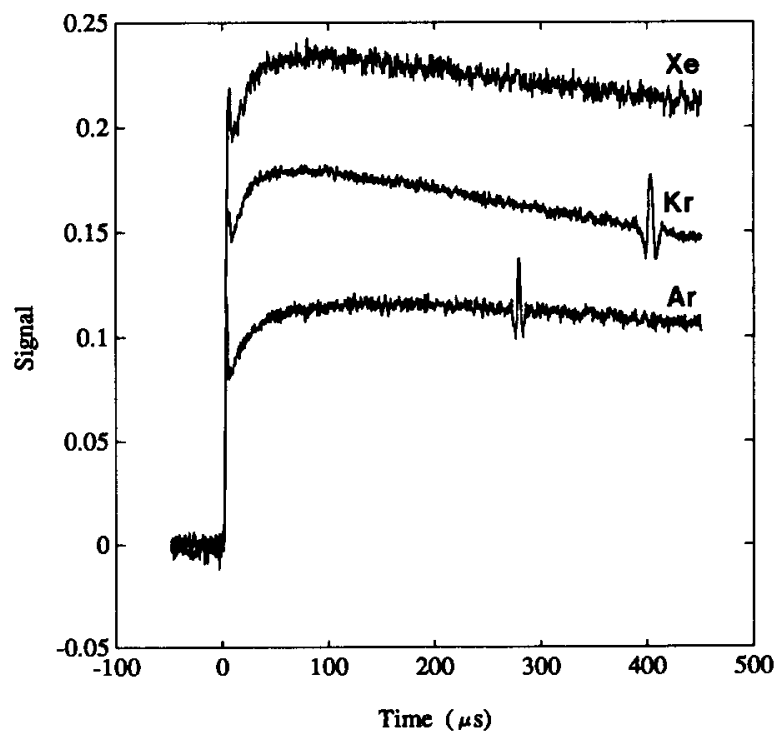

FIG. 2. Typical experimental TDTL signals for a mixture of $\mathrm{NO}_{2}(0.04$ Torr) and $\mathrm{Xe}, \mathrm{Kr}$, or $\mathrm{Ar}$ ( $\approx 200$ Torr) in order of decreasing intensity. See Table II for the physical conditions. 
TABLE I. Physical properties. $T=298 \mathrm{~K}$.

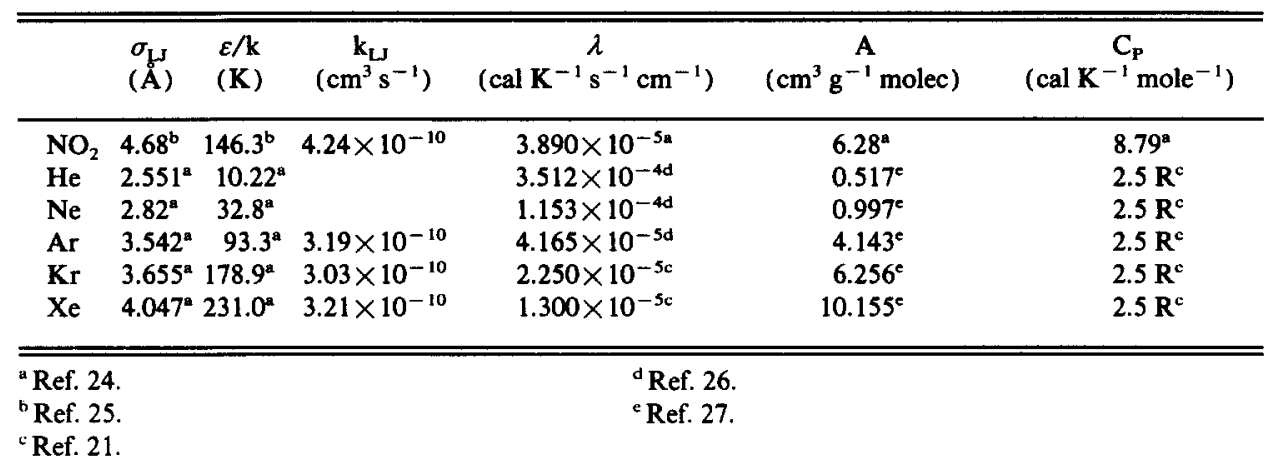

energy deposition occurs in a time scale that is shorter than the acoustic transit time; if the acoustic time were shorter, no spike would be seen. ${ }^{13}$

The small perturbations in Fig. 2 at $273 \mu$ s for Ar and at $396 \mu$ s for $\mathrm{Kr}$ are due to returning acoustic waves, which were reflected from the cell walls; a reflected acoustic wave for Xe in our cell occurs at $495 \mu \mathrm{s}$. Even if the reflected acoustic waves have no particular significance in our experiments, it is interesting to note that if the laser beams are located slightly off the cell axis, the observed acoustic wave perturbations for the first reflection (and all subsequent odd numbered reflections) tends to a focus away from the optical axis, while the second reflection (and all subsequent even numbered reflections) focuses at the original optical axis. In most experiments, only the even numbered acoustic reflections produced significant TDTL signals, due to this effect.

\section{BEAM SIZE DETERMINATION}

The characterization of the laser beam is important because the initial spatial distribution of the excited molecules is determined by the spatial distribution of the laser beam, as well as the time constants for acoustic waves, thermal conductivity and diffusion, as discussed earlier. In all the simulations reported here, the spatial profile of the laser was assumed to be Gaussian and the beam size parameter $r_{b}$ was determined on that basis from the experimental data. For a Gaussian beam, $r_{b}$ is the radius at which the intensity drops to $1 / e$ of the intensity at beam center. For highest accuracy thermal lensing measurements, very high quality Gaussian laser beams are desirable, but for most pulsed tunable dye lasers, the beams are clearly not Gaussian. Even if the laser beam is Gaussian, beam size measurements require data of high precision. Given these difficulties, it is often more practicable to determine $r_{b}$ by using a standard gas of known thermal conductivity coefficient, and calculating $r_{b}$ from the observed time for thermal conductivity. If the laser beam is non-Gaussian, $r_{b}$ loses its physical significance and merely becomes a fitting parameter.

In our experiments for each inert gas, we measured the TDTL signal at high pressures and for very long times where the decay of the lens is controlled by thermal conductivity. For these conditions, Bailey et al. ${ }^{21}$ have shown that a plot of Eq. (19) allows determination of $\tau_{c}$ for a Gaussian laser beam:

$$
\left[(1-S)^{-1 / 2}-1\right]^{-1 / 2}=\left(t+\tau_{c}\right) C,
$$

where $C$ is a constant.

Using Eq. (5), $r_{b}$ can be determined from $\tau_{c}$, provided that the thermal conductivity coefficient for the gas mixture is known. In Fig. 3 we show a plot of the left side of Eq. (19) vs time for 498 Torr of $\mathrm{Xe}$ and 0.098 Torr of $\mathrm{NO}_{2}$, and the data are well represented by Eq. (19) [from the observed decay, $r_{b}=0.072 \mathrm{~cm}$ ]. If Fig. 3 had shown some curvature at the earliest times, that may have been an indication that energy transfer is still playing a role at the long times and high pressure of the experiment. The procedure just outlined was carried out each day for pressures at which thermal conductivity was the controlling factor. Typically, beam radius for the experiments averaged $0.072 \pm 0.005 \mathrm{~cm}$.

Some simulations were carried out for assumed nonGaussian profiles. The simulated TDTL signals that resulted were plotted according to Eq. (19) and it was found that the plots were linear, despite the non-Gaussian profile. Thus, curvature in the plot is not a diagnostic for beam profile. Moreover, the assumed non-Gaussian profiles did not lead to significant differences in derived energy transfer param-

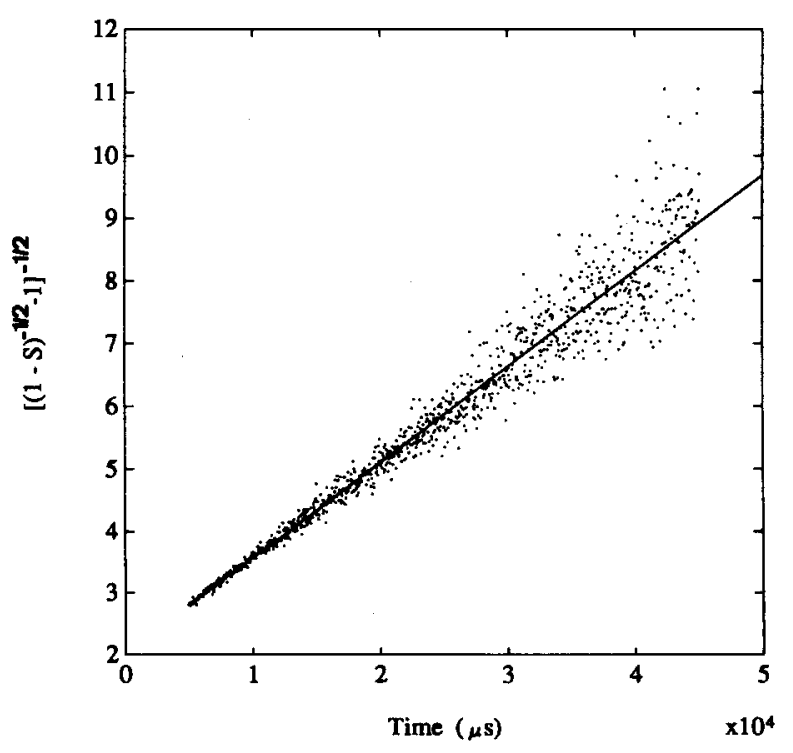

FIG. 3. Plot of $\left[(1-S)^{-1 / 2}-1\right]^{-1 / 2}$ vs time for an experiment with 498 Torr of Xe and 0.098 Torr of $\mathrm{NO}_{2}$. 
eters, and thus Gaussian profiles were assumed in all subsequent analysis.

\section{ENERGY TRANSFER RESULTS}

Figure 4 shows typical TDTL signals for several gas pressures, holding the $\mathrm{NO}_{2} / \mathrm{Kr}$ mixing ratio constant at $\sim 1 / 5000$ to minimize the effects of $\mathrm{NO}_{2}-\mathrm{NO}_{2}$ collisions. Also shown in Fig. 4 are theoretical simulations using the same time-dependent bimolecular rate constants at all the pressures, demonstrating the consistency of the results. The general shapes of the TDTL signals are very well reproduced for this particular set of energy transfer parameters, although for some of the pressures it is possible to find a better fit. Similar experiments and simulations have been carried out for Ar and Xe, using the same range of pressures and the same dilution ratio. For each of the inert collider gases it is possible to simulate all the signals with a set of energy transfer parameters characteristic of the particular collider. Also needed in the simulations are estimates of the fraction of excited molecules, which depends on the pump laser energy and the absorption cross section, which was taken to be $5 \times 10^{-19} \mathrm{~cm}^{2} .{ }^{28}$ Experiments were also carried out for larger $\mathrm{NO}_{2}$ mixing ratios in order to determine the rate constant for $\mathrm{NO}_{2}^{*}-\mathrm{NO}_{2}$ collisions. The simulation rate constants, experimental laser energy measurements, and beam size parameters $r_{b}$ that produce the best fits for $\mathrm{Ar}, \mathrm{Kr}$, and $\mathrm{Xe}$ runs are presented in Table II.

The sensitivity of the simulation parameters was deter-

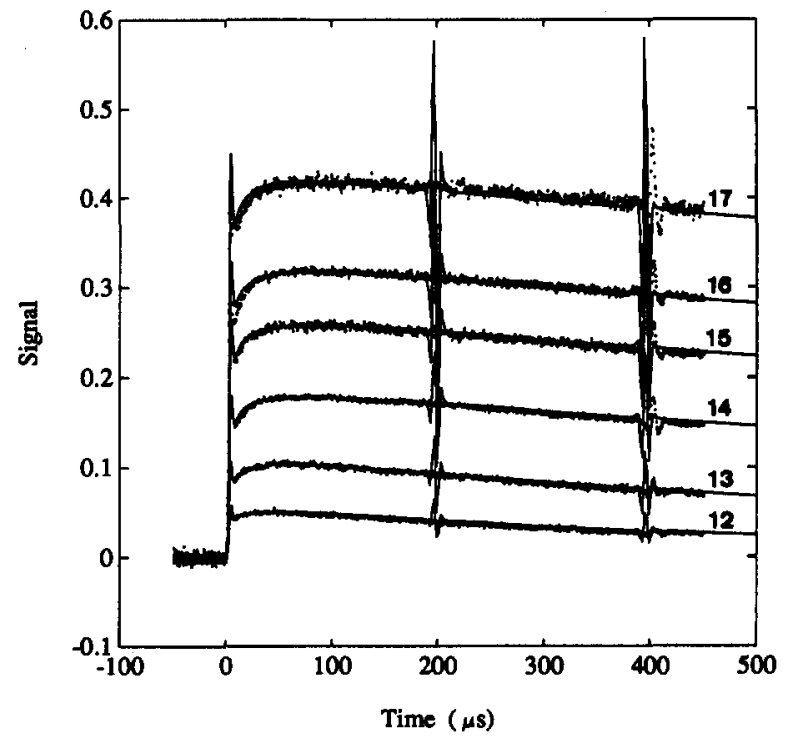

FIG. 4. Experimental and calculated TDTL signals for excited $\mathrm{NO}_{2}$ in $\mathrm{Kr}$. The simulations have been done using the energy transfer rate constant corresponding to run 12 in Table II. See Table II for the curve labels and for the physical conditions of these calculations.

mined by varying assumed values for 1 ) the beam size, 2) the values of the rate constant, and 3 ) the functional form of $k_{e}$. The effect of the beam size is particularly important in simulating the decay of the signal at low and moderate pressures,

TABLE II. Energy transfer parameters"

\begin{tabular}{|c|c|c|c|c|c|c|c|c|c|c|}
\hline \# & Gas & $P$ (Torr) & $\mathrm{NO}_{2}$ (Torr) & $k_{o}$ & $A$ & $k_{1}$ & $B$ & $k_{\operatorname{lin} \mathbf{n}}$ & $E_{\text {laser }}(\mathrm{mJ})$ & $r_{b}(\mathrm{~cm})$ \\
\hline 1 & $\mathrm{Ar}$ & 47.68 & 0.0093 & $5.82 E-13$ & 0.004 & $1.07 E-14$ & 0.00004 & $1.78 E-15$ & 2.74 & 0.0788 \\
\hline 2 & $\mathrm{Ar}$ & 99.12 & 0.019 & $5.60 E-13$ & 0.004 & $1.03 E-14$ & 0.00004 & $1.71 E-15$ & 2.80 & 0.0788 \\
\hline 3 & Ar & 199.96 & 0.039 & $5.55 E-13$ & 0.004 & $9.26 E-15$ & 0.00005 & $1.39 E-15$ & 2.96 & 0.0788 \\
\hline 4 & $\mathrm{Ar}$ & 299.94 & 0.059 & $5.55 E-13$ & 0.004 & $9.26 E-15$ & 0.00004 & $1.54 E-15$ & 3.12 & 0.0850 \\
\hline 5 & Ar & 399.92 & 0.078 & $5.55 E-13$ & 0.004 & $9.18 E-15$ & 0.00004 & $1.54 E-15$ & 3.08 & 0.0788 \\
\hline 6 & Ar & 500 & 0.098 & $5.55 E-13$ & 0.004 & $1.02 E-14$ & 0.00004 & $1.70 E-15$ & 3.08 & 0.0788 \\
\hline 7 & Ar & 38.17 & 0.079 & $6.10 E-13$ & 0.004 & $2.02 E-14$ & 0.00004 & $4.36 E-15$ & 3.64 & 0.0788 \\
\hline 8 & Ar & 14.68 & 0.079 & $6.09 E-13$ & 0.004 & $4.41 E-14$ & 0.00004 & $1.05 E-14$ & 3.68 & 0.0788 \\
\hline 9 & $\mathrm{Ar}$ & 61.72 & 0.079 & $6.00 E-13$ & 0.004 & $1.57 E-14$ & 0.00004 & $3.60 E-15$ & 3.68 & 0.0767 \\
\hline 10 & $\mathbf{K}_{\mathbf{r}}$ & 92.62 & 0.072 & $5.99 E-13$ & 0.004 & $1.73 E-14$ & 0.00004 & $2.86 E-15$ & 4.48 & 0.0767 \\
\hline 11 & $\mathrm{~K}_{\mathbf{r}}$ & 25.25 & 0.005 & $9.16 E-13$ & 0.004 & $1.22 E-14$ & 0.00004 & $2.44 E-15$ & 0.53 & 0.0670 \\
\hline 12 & $\mathbf{K r}$ & 50.48 & 0.0099 & $9.17 E-13$ & 0.004 & $1.22 E-14$ & 0.00004 & $2.44 E-15$ & 4.20 & 0.0670 \\
\hline 13 & $\mathbf{K}_{\mathbf{r}}$ & 102.46 & 0.0202 & $9.03 E-13$ & 0.004 & $1.20 E-14$ & 0.00004 & $2.41 E-15$ & 4.56 & 0.0640 \\
\hline 14 & $\mathbf{K r}$ & 198.46 & 0.0391 & $9.33 E-13$ & 0.004 & $1.24 E-14$ & 0.00004 & $2.49 E-15$ & 3.56 & 0.0670 \\
\hline 15 & $\mathbf{K r}$ & 302.94 & 0.0597 & $9.16 E-13$ & 0.004 & $1.22 E-14$ & 0.00004 & $2.44 E-15$ & 1.40 & 0.0670 \\
\hline 16 & $\mathrm{Kr}$ & 385.92 & 0.076 & $9.23 E-13$ & 0.004 & $1.23 E-14$ & 0.00004 & $2.46 E-15$ & 1.52 & 0.0670 \\
\hline 17 & $\mathrm{Kr}$ & 497 & 0.098 & $7.76 E-13$ & 0.005 & $1.24 E-14$ & 0.00004 & $2.48 E-15$ & 0.53 & 0.0670 \\
\hline 18 & $\mathrm{Xe}$ & 25.13 & 0.0049 & $9.21 E-13$ & 0.004 & $1.23 E-14$ & 0.00004 & $2.45 E-15$ & 1.92 & 0.0729 \\
\hline 19 & $\mathrm{Xe}$ & 50.23 & 0.0099 & $9.21 E-13$ & 0.004 & $1.23 E-14$ & 0.00004 & $2.46 E-15$ & 4.72 & 0.0680 \\
\hline 20 & $\mathrm{Xe}$ & 100.17 & 0.0197 & $9.24 E-13$ & 0.004 & $1.23 E-14$ & 0.00004 & $2.46 E-15$ & 1.46 & 0.0680 \\
\hline 21 & $\mathbf{X e}$ & 200.91 & 0.0395 & $9.21 E-13$ & 0.004 & $1.23 E-14$ & 0.00004 & $2.46 E-15$ & 0.70 & 0.0729 \\
\hline 22 & $\mathrm{Xe}$ & 299.89 & 0.059 & $9.26 E-13$ & 0.004 & $1.23 E-14$ & 0.00004 & $2.47 E-15$ & 0.72 & 0.0729 \\
\hline 23 & $\mathrm{Xe}$ & 402.87 & 0.0792 & $9.19 E-13$ & 0.004 & $1.23 E-14$ & 0.00004 & $2.45 E-15$ & 0.75 & 0.0729 \\
\hline 24 & $\mathrm{Xe}$ & 487.85 & 0.098 & $7.90 E-13$ & 0.005 & $1.26 E-14$ & 0.00004 & $2.53 E-15$ & 0.78 & 0.0729 \\
\hline 25 & $\mathrm{Xe}$ & 30.16 & 0.16 & $8.18 E-13$ & 0.004 & $4.24 E-14$ & 0.00004 & $1.02 E-14$ & 0.84 & 0.0650 \\
\hline 26 & $\mathrm{Xe}$ & 50.23 & 0.16 & $9.21 E-13$ & 0.004 & $2.95 E-14$ & 0.00004 & $7.25 E-15$ & 0.51 & 0.0650 \\
\hline 27 & $\mathrm{Xe}$ & 11.1 & 0.16 & $7.78 E-13$ & 0.004 & $1.17 E-13$ & 0.00004 & $2.75 E-14$ & 1.18 & 0.0700 \\
\hline 28 & $\mathrm{Xe}$ & 99.4 & 0.16 & $8.67 E-13$ & 0.0045 & $2.11 E-14$ & 0.00004 & $4.65 E-15$ & 0.40 & 0.0729 \\
\hline 29 & $\mathrm{Xe}$ & 50.57 & 0.0814 & $9.13 E-13$ & 0.004 & $2.07 E-14$ & 0.00004 & $4.81 E-15$ & 0.36 & 0.0729 \\
\hline
\end{tabular}

" $k_{c}$ in units of $\mathrm{cm}^{3} \mathrm{~s}^{-1}$. Excitation energy: $21631 \mathrm{~cm}^{-1}, T=298 \mathrm{~K}$; cell length: $89 \mathrm{~cm}$, cell radius: $2.19 \mathrm{~cm}$. 


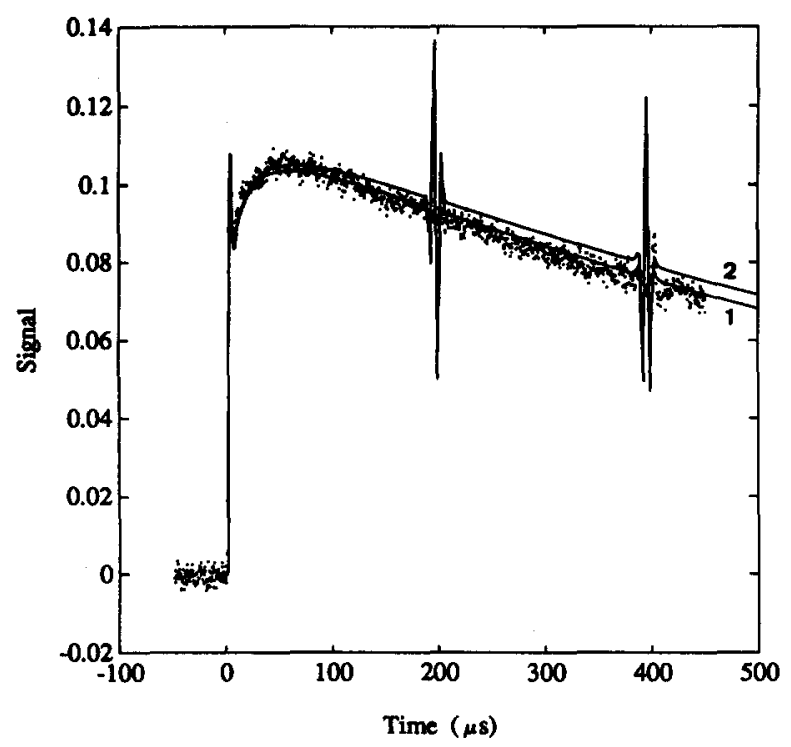

FIG. 5. Effect of a variation in the size of the laser beam on the calculated TDTL signal. 1) $\left.r_{b}=0.067 \mathrm{~cm}, 2\right) r_{b}=0.064 \mathrm{~cm}$. See Table II, run 13 for pressure conditions and energy transfer parameters.

as illustrated in Fig. 5 for Experiment 13 (see Table II for conditions). The $5 \%$ change in $r_{b}$ clearly degrades the simulation. The maximum change in $r_{b}$ necesary to produce a good fit to any of the experimental signals was only $5 \%$. At higher pressure and for time scales in which energy transfer is playing a more important role, the sensitivity to $r_{b}$ is less important.

Figures 6-8 illustrate the effects of changing the bimolecular rate constant in simulations of experiments at three different pressures. In each case, the best fit $k_{e}$ was multiplied by factors of 1.2 and 0.8 , in order to determine sensitivity to variations in the fitted rate constant. Inspection of the

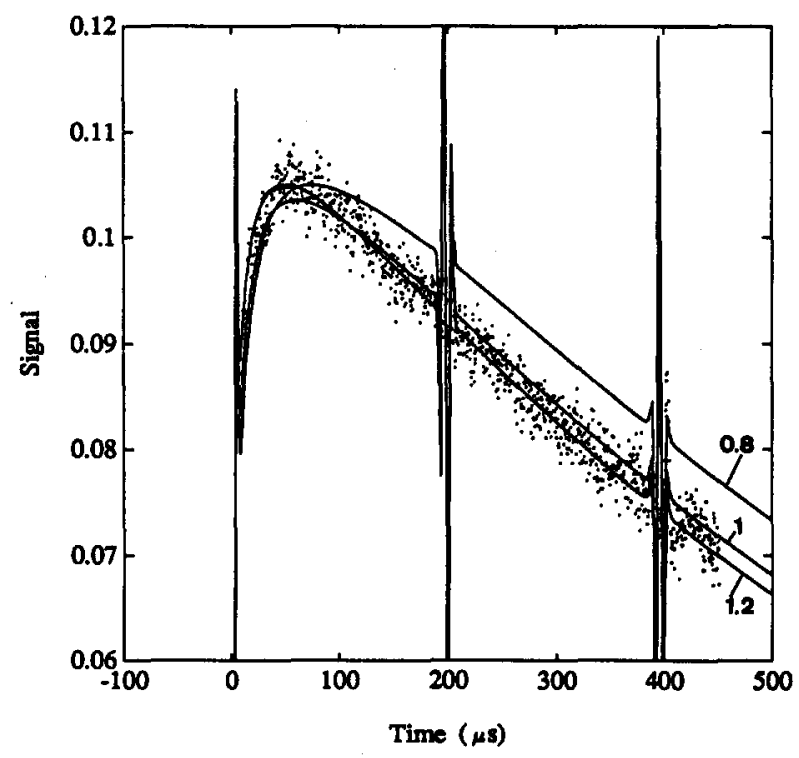

FIG. 6. Effect of a $20 \%$ change in the rate constant for energy transfer on the calculated TDTL signal for $\mathrm{NO}_{2}(0.0202 \mathrm{Torr})$ in $\mathrm{Kr}$ (102.46 Torr). The curve labels indicate the factor for which $k_{c}$, has been multiplied with respect to $k_{v}$ in Table II (run 13).

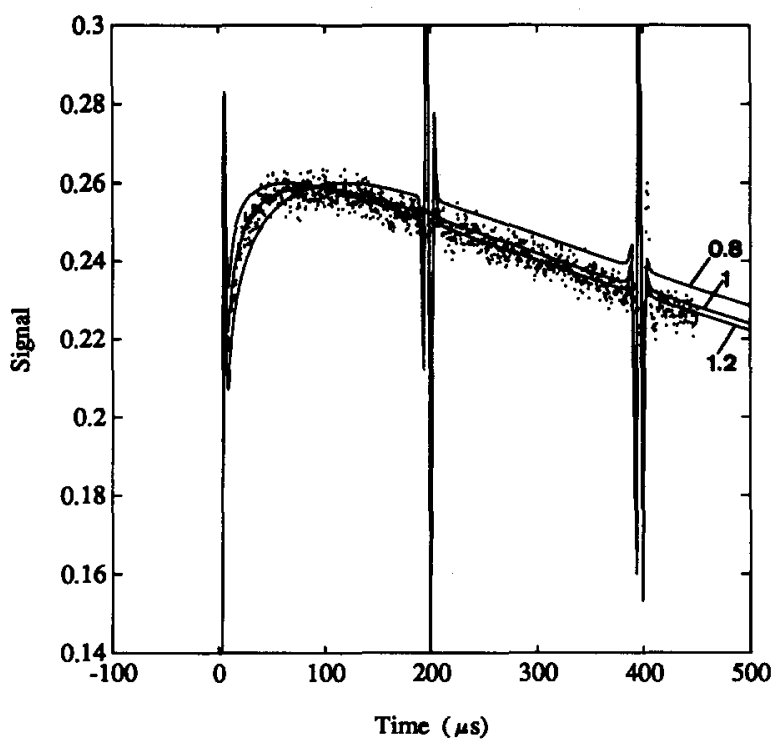

FIG. 7. The same as Fig. 6, but for 0.0597 Torr of $\mathrm{NO}_{2}$ and 302.94 Torr of Kr. Run 15 in Table II.

figures shows that the fit is very sensitive to variations in $k_{\mathrm{e}}$. For each of the three pressures, the $20 \%$ variation is the maximum change that will still produce an acceptable fit.

It is interesting to analyze in some detail how the signals are affected by the different terms in Eq. (18). The rising part of the signal and the position of the spike are controlled mostly by the first term. In particular the ratio $k_{o} / A$ controls the amount of energy that has been deposited in the time period in which the acoustic effects are competing with energy transfer. For a good fit, the ratio was found to be highly constrained.

Note that in our simulations the position of the spike has been reproduced correctly, but the magnitude of the spike in the calculated signal is in general overestimated to a small

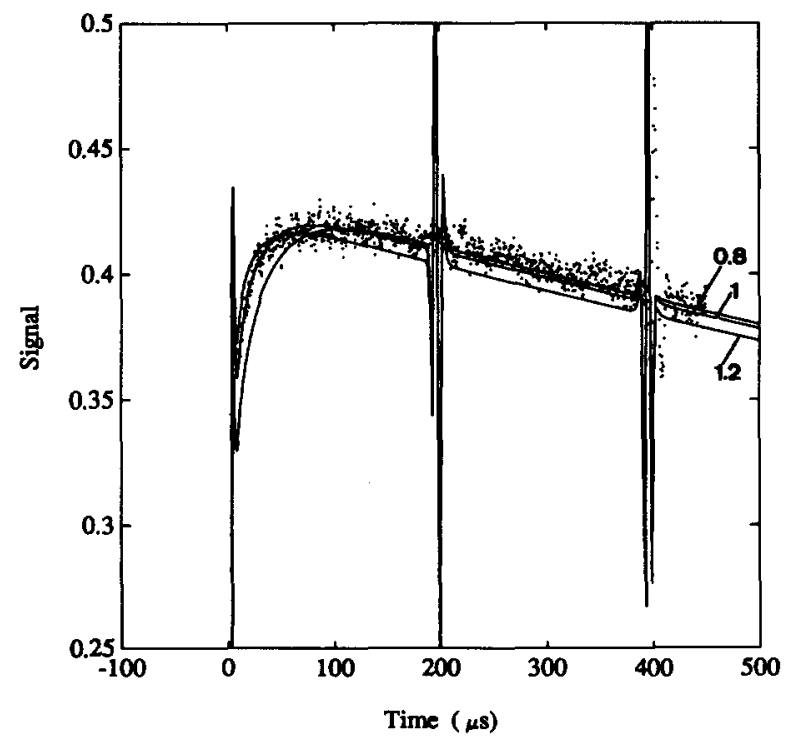

FIG. 8. The same as Fig. 6, but for 0.098 Torr of $\mathrm{NO}_{2}$ and 497 Torr of $\mathrm{Kr}$. Run 17 in Table II. 


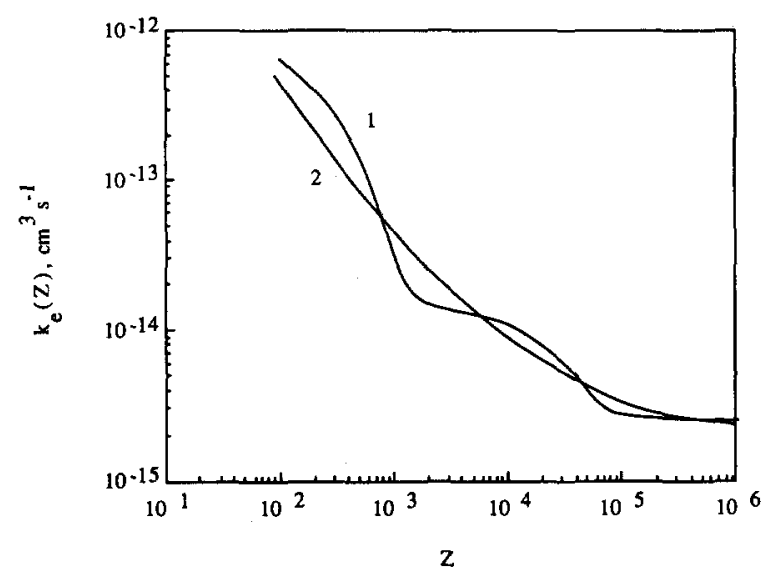

FIG. 9. Example of the functional form for the energy transfer rate constant $k_{u}(Z)$ for the deactivation of $\mathrm{NO}_{2}$ by $\mathrm{Ar}, \mathrm{Kr}$ or $\mathrm{Xe}$, in units $\mathrm{cm}^{3} \mathrm{~s}^{-1}$. (corresponding to run 14 in Table II.) 1) Eq. (18).2) Smoothed $k_{c}(Z)$; see text for details.

extent. This problem is likely due to imperfections in the shape of the laser beam, as discussed above. Following the spike, the slowly rising signal and the position of the broad maximum are controlled by the second and third terms in Eq. (18): a fast rate constant shifts the maximum to shorter times and increases the slope of the rising signal. The term that dominates will, in general, depend on the total pressure. The effects of the third term in Eq. (18) are also seen at low pressures, where the decay of the signal is controlled by this term and by the size of the laser beam.

The sensitivity of the simulations to the assumed functional form of the rate constant was also investigated. The sum of exponential factors [Eq. (18)] reproduces all the characteristics of the TDTL signal, but the rate constant exhibits a series of "steps", which may, or may not be physically significant. To determine whether the "steps" are significant, a smoother function was substituted and simulations were carried out. The smoother function was obtained by a second order polynomial least squares fit of $\ln \left(k_{e}\right)$ vs $\ln (Z)$, where $Z=\omega t$, the number of collisions, as shown in Fig. 9. Simulations carried out using the smoother version of $k_{e}$ also reproduced the major features of the TDTL signals, and thus the "steps" are an artifact due to the assumed form for $k_{c}$ and have no real significance.

TABLE III. Recommended energy transfer rate constants ${ }^{\mathrm{a}}$ and $\langle\langle\Delta E\rangle\rangle$ parameters $^{\mathrm{b}}$

\begin{tabular}{cccccc}
\hline \hline Collider & $k_{o}$ & $A$ & $k_{1}$ & $B$ & $k_{\text {lim }}$ \\
\hline $\mathrm{Ar}$ & $5.82 E-13$ & 0.004 & $1.07 E-14$ & 0.00004 & $1.78 E-15$ \\
$\mathrm{Kr}$ & $9.33 E-13$ & 0.004 & $1.24 E-14$ & 0.00004 & $2.49 E-15$ \\
$\mathrm{Xe}$ & $9.21 E-13$ & 0.004 & $1.23 E-14$ & 0.00004 & $2.46 E-15$
\end{tabular}

$k_{\mathrm{r}}\left(\right.$ in $\left.\mathrm{cm}^{3} \mathrm{~s}^{-1}\right)=k_{o} \exp (-A \omega t)+k_{1} \exp (-B \omega t)+k_{\text {lim }}$.

${ }^{\mathrm{b}}\langle\langle\Delta E(t)\rangle\rangle=k_{\mathrm{c}}(t) E(t) / \omega$ and $E(t)=H_{1} \exp \left\{\left[\left(k_{o} / A \omega\right)(\exp (-A \omega t)\right.\right.$

$\left.-1)]+\left[\left(k_{1} / B \omega\right)(\exp (-B \omega t)-1)\right]-k_{\lim } t\right\}$, where $H_{1}=21631$ $\mathrm{cm}^{-1}$. For the uncertainties in the $\langle\langle\Delta E\rangle\rangle$ values derived from these parameters, see the discussion in the text.

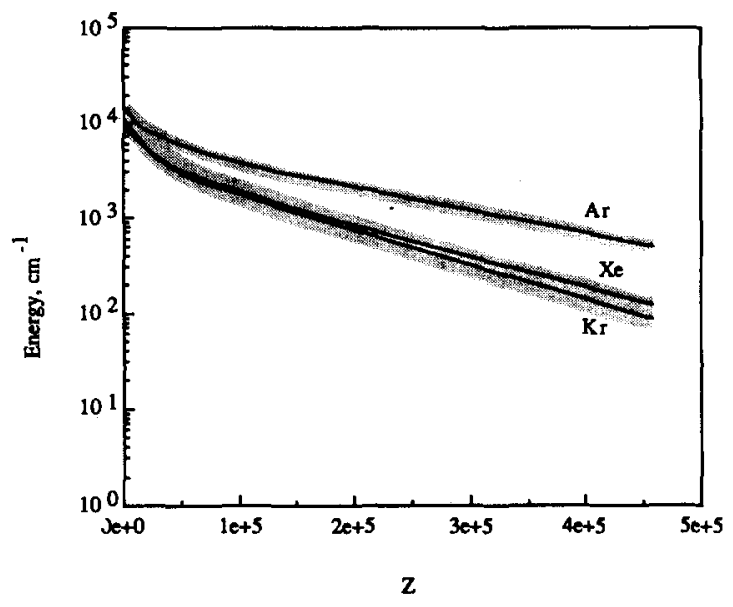

FIG. 10. Dependence of the energy content of excited $\mathrm{NO}_{2}$ with the number of collisions $(Z)$ for $\mathrm{Kr}, \mathrm{Xe}$, and Ar.

\section{DISCUSSION}

\section{Deactivation by Inert Gases}

From simulations of each experimental run in Table II, values for $k_{e}(t)$ were obtained, each with an uncertainty of $\sim 20 \%$. A careful examination of the data showed that a single "global" expression for $k_{e}(t)$ simulates the complete set of measurements for a single collider gas, if small discrepancies are accepted for the first few microseconds of the signals. This is acceptable, because the laser beams are nonGaussian and the initial gas-dynamic relaxation is not accurately described by the theory described above. The initial gas-dynamic relaxation tends to produce an axially symmetric Gaussian profile, and the simulations should become more accurate at later times. The global expressions for $k_{e}(t)$ are presented in Table III.

From Eq. (14) and the global expressions for $k_{e}(t)$, the bulk average vibrational energy $\langle\langle E(t)\rangle\rangle$ in the excited $\mathrm{NO}_{2}$ can be calculated, as shown in Fig. 10, as a function of the number of collisions $Z$ for each collider gas. The shaded area in the figure represents the estimated uncertainties in $\langle\langle E(Z)\rangle\rangle$. From Eq. (17), the time-dependent bulk average amount of energy transferred per collision $\langle\langle\Delta E(t)\rangle\rangle$ can be calculated and represented as a function of $\langle\langle E(t)\rangle\rangle$, as shown in Fig. 11, where the shaded area represents the estimated uncertainties.

In Fig. 11, the shapes of the curves and the magnitudes of $\langle\langle\Delta E\rangle\rangle$ are quite similar for the three inert gases, in general agreement with studies of other excited species where no significant differences were observed among the inert gases. ${ }^{8,9}$ Note that the "kinks" in the curves are a consequence of the function used for $k_{e}(t)$ and have no physical significance; when the smoother function for $k_{e}$ was used, the general shape of $\langle\langle\Delta E(Z)\rangle\rangle$ is the same, but the kinks are smoothed out.

The collisional deactivation of $\mathrm{NO}_{2}$ in Fig. 11 shows a very strong dependence on vibrational energy. For energies up to $\sim 4000 \mathrm{~cm}^{-1}$, the energy-dependence is near linear, but it increases to an approximate quadratic dependence from $\sim 4000 \mathrm{~cm}^{-1}$ to $\sim 10000 \mathrm{~cm}^{-1}$. In fact the whole re- 


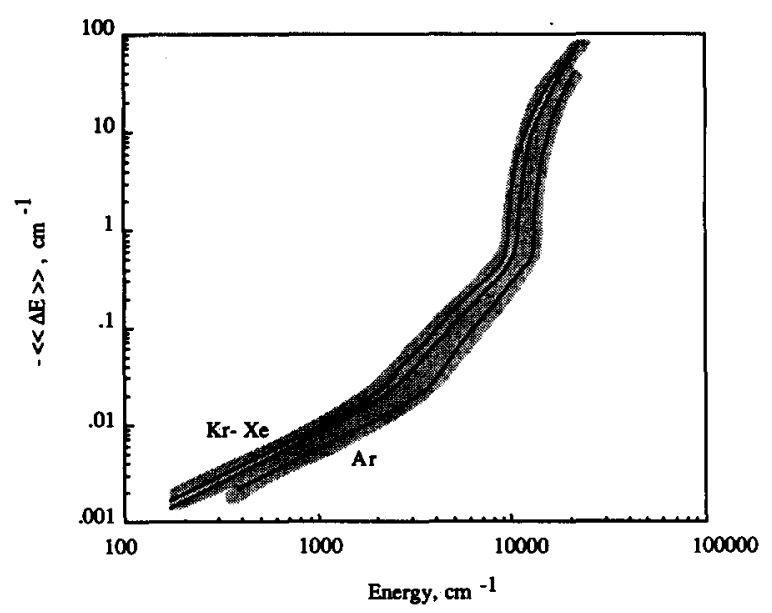

FIG. 11. Average energies $\langle\langle\Delta E\rangle\rangle$ transferred per collision of excited $\mathrm{NO}_{2}$ with $\mathrm{Kr}, \mathrm{Xe}$, and Ar. Shading shows estimated uncertainties.

gion below $10000 \mathrm{~cm}^{-1}$ could be moderately well represented by a quadratic function. At still higher energies, the results are uncertain, but it appears that the dependence is at least 5 th order, and possibly larger.

The exact position of the sharp bend is difficult to assess and this fact affects the precision of the derived $\langle\langle\Delta E\rangle\rangle$ values. For example if we take the $k_{e}$ values that produce the best fits for 200 and 500 Torr of $\mathrm{Kr}$ (see Table II) and calculate $\langle\langle\Delta E\rangle\rangle$ using Eq. (17) we find that the bend occurs at $E \sim 9500 \mathrm{~cm}^{-1}$ for 200 Torr and at $E \sim 12000 \mathrm{~cm}^{-1}$ for 500 Torr. Is clear that in this particular region the derived $\langle\langle\Delta E\rangle\rangle$ are quite different leading to a large uncertainty. $\mathbf{A}$ comparison of the results derived from the two fits indicate an uncertainty of $20 \%$ in the $\langle\langle\Delta E\rangle\rangle$ at energies close to $21600 \mathrm{~cm}^{-1}$, a factor $\sim 9$ in the region of the sharp bend, $35 \%$ at $9000 \mathrm{~cm}^{-1}, 30 \%$ at $7000 \mathrm{~cm}^{-1}, 40 \%$ at $3000 \mathrm{~cm}^{-1}$, and less than $10 \%$ at lower energies. Similar analysis for $\mathrm{Xe}$ leads to almost the same relative uncertainty in the $\langle\langle\Delta E\rangle\rangle$ values. The sharp bend for Ar occurs at $\sim 12000 \mathrm{~cm}^{-1}$ for all the fits and therefore the estimated uncertainty from this analysis is smaller than for $\mathrm{Kr}$ and $\mathrm{Xe}$. It is important to remember that if the small differences between experimental and calculated signals during the first 5-10 $\mu$ s are accepted, all the pressures can be fitted with the same $k_{e}$. As discussed above, these discrepancies are due in part to the non-Gaussian laser beam and therefore is probable that the errors in the region of $10000-12000 \mathrm{~cm}^{-1}$ are overestimated.

Extrapolations of the $-\langle\langle\Delta E\rangle\rangle$ curves to the dissociation energy, $25132 \mathrm{~cm}^{-1}$ are quite uncertain, but nonetheless the values of $\sim 60,83$, and $90 \mathrm{~cm}^{-1}$ for the colliders Ar, $\mathrm{Xe}$, and $\mathrm{Kr}$, respectively, were estimated. These values for $\langle\langle\Delta E\rangle\rangle$ can be compared with those obtained from low pressure thermal recombination rate constant, $k_{o}$, measurements. Unfortunately, such $\langle\Delta E\rangle$ values are determined from collision efficiencies, $\beta_{c}$, which are, in turn, based in theoretical calculations ${ }^{29-31}$ of the strong collider rate constant. For example, Troe and coworkers ${ }^{9}$ reported $\beta_{c}=0.46$ for $\mathrm{Ar}$ (relative to $\mathrm{C}_{3} \mathbf{F}_{\mathbf{8}}$ collider) when an extra factor of $1.7^{32}$ is included to correct for the effects of the electronic excited states. This $\beta_{c}$ value corresponds to $-\langle\Delta E\rangle=300$ $\mathrm{cm}^{-1}$; other evaluations produce even larger magnitudes for $\langle\Delta E\rangle .^{33}$ The present experimental measurements indicate that $\langle\Delta E\rangle$ magnitudes derived from the unimolecular reaction studies are significantly overestimated, probably reflecting errors in the theoretical models. One point of particular concern is the contribution of electronic excited states. ${ }^{31,32}$

A more detailed comparison can be made with nonreactive systems that have been investigated recently by physical methods. In large polyatomic systems, the energy-dependence of $\langle\langle\Delta E\rangle\rangle$ is much weaker, tending to be approximately first order in energy. ${ }^{30}$ In the triatomic systems $\mathrm{SO}_{2}{ }^{8}$ and $\mathrm{CS}_{2},{ }^{9}$ an approximate quadratic dependence was observed for deactivation by the rare gases. It is also noteworthy that the magnitudes of $\langle\langle\Delta E\rangle\rangle$ are unusually large for $\mathrm{NO}_{2}$ deactivation, especially at low energies. Thus, both the energy dependence and the magnitudes of $\langle\langle\Delta E\rangle\rangle$ for $\mathrm{NO}_{2}$ are distinctive, just as the $\mathrm{NO}_{2}$ molecular properties are distinctive.

As has been mentioned earlier, several $\mathrm{NO}_{2}$ electronic excited states are strongly coupled with the ground state. This coupling has been invoked to explain the complexity in the spectrum of $\mathrm{NO}_{2}$ and the long lifetime for the visible fluorescence. Following light absorption, $\mathrm{NO}_{2}$ is initially prepared in the ${ }^{2} B_{2}$ electronic excited state, but after a short time, or a few collisions, the system undergoes internal conversion to the electronic ground state; the subsequent collisional deactivation is dominated by the ground state. The triatomics $\mathrm{CS}_{2}$ and $\mathrm{SO}_{2}$ also have strongly coupled electronic states and they have been found to have similar behavior.

The electronic structure of $\mathrm{NO}_{2}$ has been investigated in several theoretical studies. According to Gillespie et al. ${ }^{34}$ there are three electronic excited states at energies below the dissociation energy for $\mathrm{NO}_{2}$ : the ${ }^{2} B_{2}$ state origin is near 9520 $\mathrm{cm}^{-1}$, the ${ }^{2} B_{1}$ near $13390 \mathrm{~cm}^{-1}$, and the ${ }^{2} A_{2}$ state near $14841 \mathrm{~cm}^{-1}$. Experimentally the origin of the first electronic excited state has been located at $11963 \mathrm{~cm}^{-1,35}$ more than $2400 \mathrm{~cm}^{-1}$ higher than the calculated origin, but there is no question that around $10000-12000 \mathrm{~cm}^{-1}$ the system changes dramatically. Gillespie's calculations show that $\mathrm{NO}_{2}$ is strongly coupled vibronically due to the fact that the ground and excited states have different bond angles, resulting in a large vibrational-overlap Franck-Condon factor. In this case, and even if the density of states is rather low (sparse case, according to Bixon and Jortner ${ }^{36}$ ), extensive mixing can still take place. It is likely that the TDTL energy transfer results are closely related with this fact.

To understand qualitatively the sharp bend in $\Delta E$ around $10000-12000 \mathrm{~cm}^{-1}$, it is useful to consider the electronic energy as a function of bond angle for the low-lying electronic states of $\mathrm{NO}_{2}$ (Fig. 12) ${ }^{34}$ At the initial energy of our experiment $\left(21631 \mathrm{~cm}^{-1}\right)$, the amplitude of the motion can be very large (broken line in Fig. 12). Due to the strong coupling, it is likely that the system can cross freely from one potential energy surface to another, without significant trapping on any one surface. The large amplitude of vibration probably produces a very low frequency of the motion, which will favor $V-T$ energy transfer. Predictions of SSH theory indicate that the lower the frequency, the higher the 


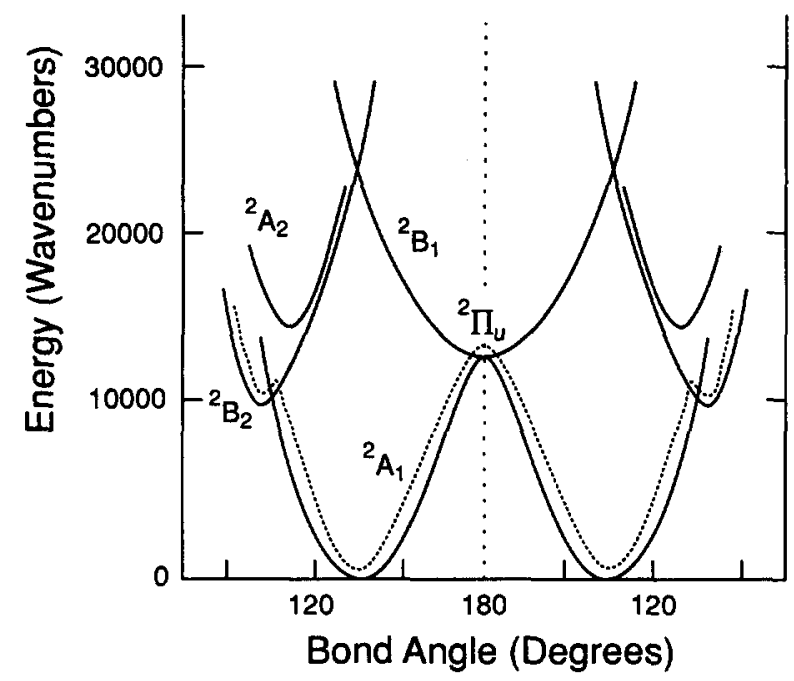

FIG. 12. Schematic of the electronic energy as a function of bond angle for the low-lying electronic states of $\mathrm{NO}_{2}$ (adapted from Ref. 34).

probability for deactivation, because the collision will provide more Fourier components at low vibrational frequencies. ${ }^{37}$ As the system energy is reduced, the $\mathrm{NO}_{2}$ bending motion becomes more and more restricted, until below the energy of the first electronic excited state the motion is confined to the lowest potential energy surface. The other internal degrees of freedom are also likely to exhibit larger amplitude motions at high energy than at low energy, due to the same effects. Thus, the probability of $V-T$ transfer will decrease markedly at lower energies. Quantitative estimates of this effect will require accurate potential surfaces and good methods for treating the couplings among the excited states.

Near the end of the energy cascade, the remaining excited $\mathrm{NO}_{2}$ is expected to reside mostly in the $(010)$ state, and the corresponding energy transfer can be compared with $V$ $T$ energy transfer measured, for example, in ultrasonic attenuation experiments. At low energy, the observed $k_{e}(t)$ becomes a constant $\left(k_{\mathrm{lim}}\right)$ and $\langle\langle\Delta E\rangle\rangle$ becomes a linear function of ensemble average energy, consistent with this view. If we equate the rate of energy deposition from our experiments to that expected from $V-T$ transfer involving the $(010)$ state, we can write

$$
\frac{d q}{d t}=-\frac{d}{d t}\langle\langle E\rangle\rangle=k_{V T} N^{*} E_{010},
$$

where $k_{V T}$ is the $V-T$ rate constant from the (010) state, $E_{010}$ is the energy of the state, and $N^{*}$ is the population in the state. The ensemble average energy in the (010) state is just

$$
\langle\langle E\rangle\rangle_{010}=N^{*} E_{010}
$$

If we assume that all of the excited molecules near the end of the energy cascade are in the (010) state, then $N^{*}=N_{1}$. Using Eq. (14), we obtain

$$
k_{V T}=\frac{H_{1}}{E_{010}} k_{\text {lim }} \text {. }
$$

The energy ratio in Eq. (22) is $21631 / 750=28.8$. Thus the $V-T$ rate constant for deactivation of the $(010)$ state is estimated to be $\sim(5.1 \pm 1.0) \times 10^{-14} \mathrm{~cm}^{3} \mathrm{~s}^{-1}$ for argon. This rate constant corresponds to a total number of collisions

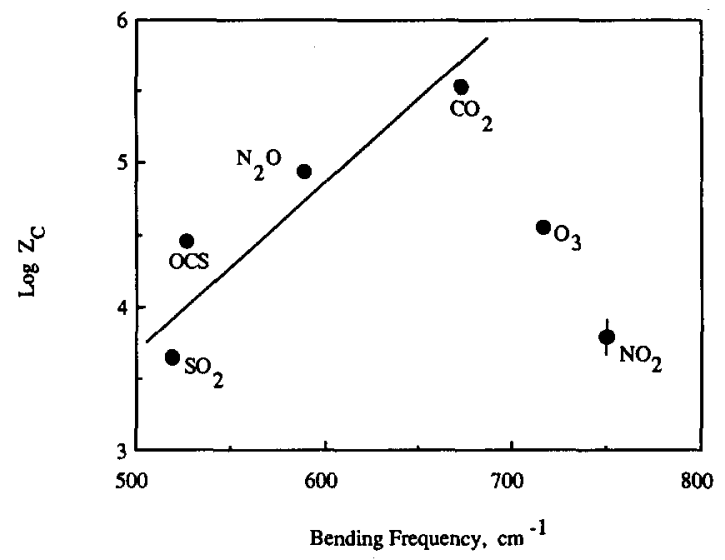

FIG. 13. Lambert-Salter plot for Ar- triatomic molecules: $\mathrm{NO}_{2}$ (this work), $\mathrm{O}_{3}$ (Ref. 44), $\mathrm{CO}_{2}$ (Ref. 45), $\mathrm{N}_{2} \mathrm{O}$ (Ref. 45), OCS (Ref. 46), and $\mathrm{SO}_{2}$ (Ref. 47).

needed for deactivation:

$$
Z_{C}=k_{\mathrm{LJ}} / k_{V T}=6200 \text {. }
$$

In Fig. $13, Z_{C}$ is compared with corresponding values for deactivation by argon of several other triatomics, and it is clear that the $\mathrm{NO}_{2}$ rate constant is unusually large in magnitude. A possible explanation for this anomalous behavior is related to the fact that $\mathrm{NO}_{2}$ is a free radical and each energy level is split into two spin components. As discussed by Nikitin,,$^{38}$ a possible cause for the high efficiency of the vibrational relaxation of NO and some other molecules with incompletely filled electron shells is the nonadiabaticity of vibrational excitation during collisions. He pointed out that when one or both colliding partners are in a degenerate electronic state, intermolecular interactions lift the electronic degeneracy, so that when molecules approach, there arise a whole set of adiabatic potential energy surfaces which are strongly coupled. Two such states are shown schematically in Fig. 14 (adapted from Nikitin's work). Nonadiabatic transitions provide additional paths of energy transfer that can greatly enhance the $V-T$ energy transfer cross section. Molecules approaching on the solid curve corresponding to $v=1$ can reach an inner turning point and be reflected, only

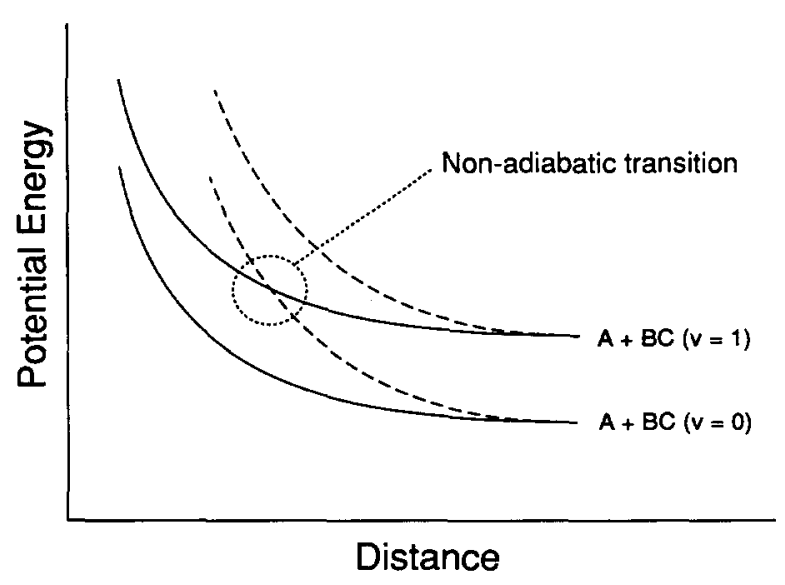

FIG. 14. Schematic of potential energy vs distance for an open shell molecule (adapted from Ref. 38 ). 
to encounter the nonadiabatic transition indicated in the figure, and switch to the $v=0$ potential curve. This mechanism is present at all energies for $\mathrm{NO}_{2}$, and at higher energies its effect is combined with that due to the large amplitude vibrational motion to produce the very large magnitudes of $\langle\langle\Delta E\rangle\rangle$ observed.

\section{Deactivation by unexcited $\mathrm{NO}_{2}$}

By varying the mixing ratio in the $\mathrm{NO}_{2}-\mathrm{Ar}$ and $\mathrm{NO}_{2}-\mathrm{Xe}$ systems, $\langle\langle\Delta E\rangle\rangle$ can be estimated for $V-T$ energy transfer from excited $\mathrm{NO}_{2}$ to unexcited $\mathrm{NO}_{2}$, and the results are shown in Fig. 15. These results may be uncertain by as much as a factor 2, nonetheless is possible to make some interesting comparisons with other studies involving $\mathrm{NO}_{2}^{*}-\mathrm{NO}_{2}$ energy transfer. Kaufman ${ }^{20}$ and coworkers, in their low pressure fluorescence studies at excitation energies ranging from 18800 to $24700 \mathrm{~cm}^{-1}$, estimated the step size for the average energy transferred per collision from a model calculation: $\langle\langle\Delta E\rangle\rangle=-1000 \pm 500 \mathrm{~cm}^{-1}$ for $\mathrm{NO}_{2}^{*}-\mathrm{NO}_{2}$ collisions at $E=18365 \mathrm{~cm}^{-1}$. Even larger values were reported by Schwartz and Johnston ${ }^{39}$ in similar experiments. From our measurements we calculate $\langle(\Delta E)\rangle=-1040 \mathrm{~cm}^{-1}$ at $E=18365 \mathrm{~cm}^{-1}$, in good agreement with the fluorescence studies, especially considering the uncertainties in the respective measurements. Since the TDTL technique is only sensitive to $V-T$ energy transfer and the fluorescence experiments measure total energy transfer, the good agreement between the two techniques may be an indication that $V-T$ transfer is one of the most important paths for highly excited $\mathrm{NO}_{2}$. This conclusion is consistent with the recent finding of inefficient $V-V$ energy transfer from excited $\mathrm{NO}_{2}$ to $v_{3}$ of $\mathrm{CO}_{2}{ }^{40}$

The conclusion that $V-T$ transfer is a major pathway for deactivation in $\mathrm{NO}_{2}^{*}-\mathrm{NO}_{2}$ collisions is somewhat surprising at first glance, because $\mathrm{NO}_{2}$ can react to produce $\mathrm{N}_{2} \mathrm{O}_{4}$, which is weakly bound chemically. One might expect the chemically bound $\mathrm{N}_{2} \mathrm{O}_{4}^{*}$ to have a lifetime long enough before redissociation for vibrational energy to completely randomize and be shared equally between the two $\mathrm{NO}_{2}$ molecules after they have separated. If this model is correct, the

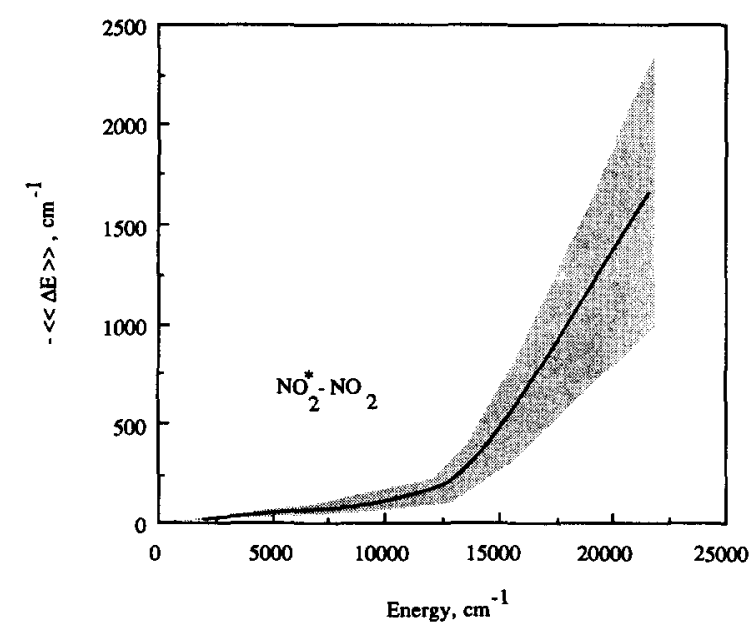

FIG. 15. The same as Fig. 11, but for $\mathrm{NO}_{2}$ collider gas. probability of forming a "strong complex" is given by the ratio of $k_{\text {rec }} / k_{\mathrm{LJ}}$, where the recombination rate constant is at the high pressure limit. If each "strong complex" results in half the excitation energy being lost during each collision (complete energy randomization) then the bulk average energy transferred per collision is given by

$$
-\langle\langle\Delta E\rangle\rangle_{\text {model }}=\frac{1}{2} \frac{k_{\mathrm{rec}}}{k_{\mathrm{LJ}}}\langle\langle E\rangle\rangle \text {. }
$$

Taking $k_{\text {rec }}$ from the work of Cobos and Troe, ${ }^{41}$ $\langle\langle\Delta E\rangle\rangle_{\text {model }} \approx-18 \mathrm{~cm}^{-1}$, when $\langle\langle E\rangle\rangle=18365 \mathrm{~cm}^{-1}$. This energy transfer would be mostly $V-V$ transfer, which does not produce TDTL signals. This quantity is small, compared with the total $\langle\langle\Delta E\rangle\rangle$ observed in both the fluorescence quenching experiments and the TDTL measurements, and therefore is consistent with the majority of deactivation occurring through $V-T$ energy transfer.

It is also possible to compare our results for the $\mathrm{NO}_{2}^{*}-\mathrm{NO}_{2}$ deactivation with a model calculation presented by Adler-Golden ${ }^{42}$ to interpret the $\mathrm{NO}_{2}$ chemiluminescence. He assumed a coarse-grained stepladder model with energy levels separated by $1000 \mathrm{~cm}^{-1}$. By solving a system of linear equations he evaluated the rate constant $k_{M}(i, j)$ for collisional energy transfer from the grain $i$ to $j$, this rate constant can be related to $\langle\langle\Delta E\rangle\rangle$ using the relation

$$
\langle\langle\Delta E\rangle\rangle=\frac{k_{M}(i, j)}{k_{\mathrm{LJ}}} \times 1000 \mathrm{~cm}^{-1}
$$

The derived $\langle\langle\Delta E\rangle\rangle$ values are about a factor of three smaller than our experimental values for energies in the range from 5000 to $21000 \mathrm{~cm}^{-1}$, but at $1000 \mathrm{~cm}^{-1}$, the difference is only $\sim 20 \%$.

In recent infrared fluorescence experiments, McAndrew et al. ${ }^{10}$ measured collisional energy transfer involving the asymmetric stretching mode of $\mathrm{NO}_{2}$ subsequent to excitation with a tunable dye laser. Unfortunately, the infrared intensities depend on unknown populations in the specific $\mathrm{NO}_{2}$ excited states, and so it was impossible to make a detailed quantitative analysis of the results. Despite the difficulties, McAndrew et al. reached the conclusion that the energy-transfer rate probably exhibits an energy-dependence. Their conclusion is consistent with the results obtained in the present work, although a detailed comparison is not possible.

\section{CONCLUSIONS}

In this work we have presented a detailed study of $V-T$ energy transfer in excited $\mathrm{NO}_{2}$. The results show that $\langle\langle\Delta E\rangle\rangle$ for this system exhibits a very strong energy dependence of the average energy transferred per collision, even stronger than the quadratic dependence observed for $\mathbf{C S}_{2}$ and $\mathrm{SO}_{2}$. Moreover, $\langle\langle\Delta E\rangle\rangle$ changes dramatically at energies near the origins of the excited electronic states. This behavior can be explained as due to enhancement of $V-T$ transfer by the large amplitude vibrational motions associated with the coupled electronic states. The $\langle\langle\Delta E\rangle\rangle$ values at low energy were related to the $V-T$ energy transfer rate constant for deactivation of the (010) state, and the large magnitude of this rate constant can be explained by Nikitin's 
model for nonadiabatic couplings in systems with degenerate electronic states.

An important strength of the TDTL technique is that the complex spectroscopy associated with $\mathrm{NO}_{2}$ can be avoided. It would be of value to compare the results obtained here with those obtained using other techniques, however, in order to identify possible systematic errors.

A very useful adjunct to the present work would be trajectory calculations, perhaps similar to those for $\mathrm{CS}_{2}{ }^{43}$ This will require developing methods for dealing with multiple coupled electronic states and nonadiabatic transitions, in order to describe $\mathrm{NO}_{2}$ realistically.

\section{ACKNOWLEDGMENTS}

This work was funded by the U.S. Department of Energy, Office of Basic Energy Sciences. B.M.T. thanks CONICET of Argentina for a postdoctoral fellowship, and T. L. W thanks NSF for an undergraduate fellowship as part of the Research Experiences for Undergraduates program in the Department of Atmospheric, Oceanic, and Space Sciences (Grant ATM-8712631). Thanks also go to S. J. Jacobs for providing a copy of the program DIFCOLL and for discussions and assistance with the solution of the hydrodynamic equations.

'J. Shi and J. R. Barker, J. Chem. Phys. 88, 6219 (1988).

${ }^{2} J$. Shi, D. Bernfeld, and J. R. Barker, J. Chem. Phys. 88, 6211 (1988).

${ }^{3}$ J. M. Zellweger, T. C. Brown, and J. R. Barker, J. Chem. Phys. 83, 6261 (1985).

${ }^{4}$ M. L. Yerram, J. D. Brenner, K. D. King, and J. R. Barker, J. Phys. Chem. (to be published).

${ }^{5}$ H. Hippler, J. Troe, and J. Wendelken, J. Chem. Phys. 78, 6709, 6718 (1983).

${ }^{6} \mathrm{H}$. Hippler, L. Lindemann, and J. Troe, J. Chem. Phys. 83, 3906 (1985).

'B. Abel, B. Herzog, H. Hippler, and J. Troe, J. Chem. Phys. 91, 900 (1989).

${ }^{8}$ M. Heymann, H. Hippler, D. Nahr, H. J. Plach, and J. Troe, J. Phys. Chem. 92, 5507 (1988).

9J. E. Dove, H. Hippler, and J. Troe, J. Chem. Phys. 82, 1907 (1985); M. Heymann, H. Hippler, H. J. Plach, and J. Troe, J. Chem. Phys. 87, 3867 (1987).

${ }^{10}$ J. J. F. McAndrew, J. M. Preses, R. E. Weston, and G. W. Flynn, J. Chem. Phys. 90, 4772 (1989).

${ }^{11}$ F. R. Grabiner, D. R. Siebert, and G. W. Flynn, Chem. Phys. Lett. 17, 189 (1972); D. R. Siebert, F. R. Grabiner, and G. W. Flynn, J. Chem. Phys. 60, 1564 (1974)

${ }^{12}$ J. R. Barker and T. Rothem, Chem. Phys. 68, 331 (1982); P. L. Trevor, T. Rothem, and J. R. Barker, ibid. 68, 341 (1982).

${ }^{13}$ J. R. Barker and B. M. Toselli, Photothermal Investigations of Solids and Fluids, edited by Jeffrey A. Sell (Academic, New York, 1989), Chap. V.

${ }^{14}$ R. T. Bailey, F. R. Cruickshank, D. Pugh, and K. Middleton, J. Chem. Soc. Faraday II 81, 255 (1985); R. T. Bailey, F. R. Cruickshank, R. Guthrie, D. Pugh, and I. J. M. Weir, Chem. Phys. 114, 411 (1987).
${ }^{15}$ N. Presser, J. R. Barker, and R. J. Gordon, J. Chem. Phys. 78, 2163 (1983); K. M. Beck, A. Ringwelski, and R. J. Gordon, Chem. Phys. Lett. 121, 529 (1985); K. M. Beck and R. J. Gordon, J. Chem. Phys. 89, 5560 (1988).

${ }^{16}$ T. J. Wallington, M. D. Scheer, and W. Braun, Chem. Phys. Lett. 138, 538 (1987).

${ }^{17}$ H. Sontag, A. C. Tam, and P. Hess, J. Chem. Phys. 86, 3950 (1987).

${ }^{18}$ D. K. Hsu, D. M. Monts, and R. N. Zare, in Spectral Atlas Of Nitrogen Dioxide 5530 to $6480 \AA$ A (Academic, New York, 1978).

${ }^{19}$ A. E. Douglas, J. Chem. Phys. 45, 1007 (1966).

${ }^{20}$ L. F. Keyser, S. Z. Levine, and F. Kaufman, J. Chem. Phys. 54, 355 (1971); V. M. Donnelly and F. Kaufman, J. Chem. Phys. 66, 4100 (1977); V. M. Donnelly, D. G. Keil, and F. Kaufman, J. Chem. Phys. 71, 659 (1979).

${ }^{21}$ R. T. Bailey, F. R. Cruickshank, D. Pugh, and W. Johnstone, J. C. S. Faraday II, 76, 633 (1980); R. T. Bailey, F. R. Cruickshank, R. Guthrie, D. Pugh, and I. J. M. Weir, Mol. Phys. 48, 81 (1983); R. T. Bailey, F. R. Cruickshank, D. Pugh, A. McLeod, and W. Johnstone, Chem. Phys. 68, 351 (1982).

${ }^{22}$ S. J. Jacobs, Chem. Phys. 132, 71 (1989).

${ }^{23} \mathrm{M}$. Born and E. Wolf, Principles of Optics (Pergamon, New York, 1975), p. 88.

${ }^{24}$ R. C. Reid and T. K. Sherwood, The Properties of Gases and Liquids (McGraw-Hill, New York, 1966)

${ }^{25}$ J. Troe, J. Chem. Phys. 66, 4758 (1977).

${ }^{26}$ C. L. Yaws, Physical Properties (McGraw-Hill, New York, 1978).

${ }^{27}$ E. A. Moelwyn-Hughes, Physical Chemistry (Pergamon, Oxford, 1961), p. 383.

${ }^{28}$ A. M. Bass, A. E. Ledford, and A. H. Laufer, J. Res. Nat. Bur. Stand. 80A, 143 (1976).

${ }^{29}$ J. Troe, J. Chem. Phys. 66, 4745 (1977).

${ }^{30}$ I. Oref and D. C. Tardy, Chem. Rev. (to be published).

${ }^{31}$ B. M. Toselli and J. R. Barker, J. Chem. Phys. 91 , 2239 (1989).

${ }^{32}$ I. W. M. Smith, Int. J. Chem. Kinet. 16, 423 (1984).

${ }^{33}$ H. Hippler, C. Schippert, and J. Troe, Int. J. Chem. Kinet. Simp. 1, 27 (1975).

${ }^{34}$ G. D. Gillespie, A. U. Khan, A. C. Wahl, R. P. Hosteny, and M. Krauss, J. Chem. Phys. 63, 3425 (1975); G. D. Gillespie and A. U. Khan, J. Chem. Phys. 65, 1624 (1976).

${ }^{35}$ J. C. D. Brand, W. H. Chan, and J. L. Hardwick, J. Mol. Spectrosc. 65, 249 (1977).

${ }^{36}$ M. Bixon and J. Jortner, J. Chem. Phys. 50, 3284 (1969).

${ }^{37}$ J. T. Yardley, Introduction to Molecular Energy Transfer (Academic, New York, 1980).

${ }^{38}$ E. E. Nikitin, Opt. Spectrosc. 9, 8 (1960); E. E. Nikitin and S. Y. Umanski, Faraday Discussions Chem. Soc. 53, 7 (1972); V. N. Kondratiev and E. E. Nikitin, Gas-Phase Reactions (Springer, Berlin, 1981).

${ }^{39}$ S. E. Schwartz and H. S. Johnston, J. Chem. Phys. 51, 1286 (1969).

${ }^{40}$ J. Z. Chou, S. A. Hewitt, J. F. Hershberger, B. B. Brady, G. B. Spector, L. Chia, and G. W. Flynn, J. Chem. Phys. 91,5392 (1989).

${ }^{41}$ C. J. Cobos and J. Troe, J. Chem. Phys. 83, 1010 (1985).

${ }^{42}$ S. M. Adler-Golden, J. Phys. Chem. 93, 684, 691 (1989).

${ }^{43}$ M. Bruehl and G. C. Schatz, J. Chem. Phys. 89, 770 (1988); J. Phys. Chem. 92, 7223 (1988).

${ }^{44}$ J. I. Steinfeld, S. M. Adler-Golden, and J. W. Gallagher, J. Phys. Chem. Ref. Data 16, 911 (1987).

${ }^{45}$ R. T. Bailey and F. R. Cruickshank, Specialist Periodical Reports 3, 109 (1978).

${ }^{46}$ D. R. Siebert and G. W. Flynn, J. Chem. Phys. 64, 4973 (1976).

${ }^{47}$ F. Douglas Shields, J. Chem. Phys. 46, 1063 (1967). 